\title{
The relationship between low-birth weight and nephrotic syndrome in children
}

\author{
Negin Rezavand ${ }^{1}$, Abolhassan Seyedzadeh ${ }^{2}$, Mohammad-Reza Tohidi² ${ }^{*}$, Mohammad-Saleh Seyedzadeh $^{2}$, \\ Sara Hookary ${ }^{2}$, Alireza Abdi ${ }^{3}$
}

${ }^{1}$ Department of Gynecology and Obstetrics, Kermanshah University of Medical Sciences, Kermanshah, Iran ${ }^{2}$ Urology and Nephrology Research Center, Kermanshah University of Medical Sciences, Kermanshah, Iran ${ }^{3}$ Nursing and Midwifery School, Kermanshah University of Medical Sciences, Kermanshah, Iran

\section{A R T I C L E I N F O}

Article Type:

Brief Communication

\section{Article History:}

Received: 20 August 2017

Accepted: 14 November 2017

ePublished: 6 December 2017

Keywords:

Idiopathic nephrotic syndrome

Low-birth weight

Glomerular development

\begin{abstract}
A B S T R A C T
Introduction: The body weight of an infant is one of the most important factors that influences its survival, growth and development. Many clinical studies have shown a higher risk of an aggravated course of renal disease in children born with low- birth weight (LBW), due to reduction in glomerular number and development. However, there are limited clinical studies about this relationship.

Objectives: This study was conducted to evaluate the relationship between LBW and risk of idiopathic nephrotic syndrome (INS) in children.

Patients and Methods: In this case-control study, we evaluated nephrotic syndrome patients who were referred to Imam Raza hospital. Seventy patients with nephrotic syndrome as case group and 140 healthy children under age 16 with normal birth weight as control group were enrolled in the study. Data including age, sex, and birth weight were collected from patient's records.

Results: This study revealed that the risk of nephrotic syndrome in patients with LBW is two times higher than those with normal birth weight. However, there was no statistically significant difference $\left(\chi^{2}=1.58, P=0.12\right)$

Conclusion: Although the result of this study cannot show a statistically significant relationship between INS and LBW, the risk of nephrotic syndrome was twice in the case group.
\end{abstract}

Implication for health policy/practice/research/medical education:

The result of this study can improve the practice and approach of clinicians towards patients with idiopathic nephrotic syndrome (INS) and the relation between birth weight and INS.

Please cite this paper as: Rezavand N, Seyedzadeh A, Tohidi MR, Seyedzadeh MS, Hookary S, Abdi A. The relationship between low-birth weight and nephrotic syndrome in children. J Nephropharmacol. 2017;7(1):6-9. DOI: 10.15171/npj.2018.03.

\section{Introduction}

Nephrotic syndrome is defined by heavy glomerular proteinuria leading to hypoalbuminemia, hypercholesterolemia and edema which is considered as a common chronic illness in childhood (1). Most children with idiopathic nephrotic syndrome (INS) are affected by minimal change disease (MCD) and only a small proportion of patients show focal segmental glomerulosclerosis and mesangial proliferation $(1,2)$.

Over $90 \%$ of children with INS may respond to steroid treatment. However, around $60-80 \%$ of children with MCD experience relapses, and about $40 \%$ will have more than five relapses. Corticosteroids are administered for treatment of the initial episode and subsequent relapses
(3). Complications of MCD and corticosteroid therapy may be serious, especially if the clinical course shows frequent relapses with the need for prolonged use of corticosteroids. Recent investigations have focused on determining potential risk factors that could predict such an unfavorable course of MCD in children, however no suitable prediction is available yet $(1,3)$.

The application of parameters to predict the subsequent clinical course and prognosis in children with MCD may offer therapeutic implications $(3,4)$. Todays, there is little success in correlating the subsequent clinical course of nephrotic syndrome with clinical and laboratory characteristics at the onset $(3,5)$. The best predictive factors have been established as; age at onset, number of relapses 
during the first six months and initial response to steroid therapy $(3,4)$. Therefore, evaluation of other factors that aggravated the course of MCD is very valuable.

Among such factors, low-birth weight (LBW) $(<2500$ $\mathrm{g}$ ), has been shown to increase the risk of developing diseases later in life, such as obesity, insulin resistance, cardiovascular disease, hypertension and progressive kidney injury probably due to lower nephron proportion $(2,5)$. The kidneys appear to be susceptible to intrauterine growth retardation (IUGR) and are often found small in proportion to body weight. Several studies in animals and humans have described a reduced proportion of nephrons after IUGR $(6,7)$. The reduced number of nephrons results in an inborn decreased glomerular filtration surface area, while renal blood flow per glomerulus is increased in an attempt to maintain a normal overall glomerular filtration rate. In fact, hyperfiltration leads to glomerular hypertension and hypertrophy, which causes systemic hypertension and glomerular damage resulting to albuminuria and glomerulosclerosis $(1,7,8)$. Therefore, LBW can eventually lead to impairment of renal function and seems to have an association with the course of MCD $(4,8)$. Additionally, it has been shown that LBW is associated with more frequent cases of glomerular disease relapses (2).

\section{Objectives}

This study was conducted to evaluate the relationship between birth weight and INS.

\section{Patients and Methods}

This case-control study was conducted on patients who referred to the pediatric nephrology clinic, Imam-Reza hospital of Kermanshah, Iran. The samples included patients under 16 years of age who were referred to the nephrology clinic from 2014 to 2016 . Around, 70 patients with INS were enrolled as the case group. Case group was compared with 140 patients with no kidney involvement who were matched one by one for age and sex as the control group. Patients with renal dysfunction, genetic disorder, congenital heart disease and pyelonephritis were excluded from the study.

A checklist which included some information about age, gender and birth weight and duration of disease was applied.

\section{Ethical issues}

The research followed the tenets of the Declaration of Helsinki and its later amendments. Patients gave their written and informed consent to participate in this study by completing the consent form. This research approved by the university (thesis\# 92251).

\section{Statistical analysis}

After data collection, they were analyzed by using descriptive statistics. Number and gender of participants were expressed as frequency and percentage. Also, the age of participants was expressed in terms of mean and standard deviation. Fisher's exact test was used to study the relationship between birth weight and risk of nephrotic syndrome. For predicting the risk of INS by birth weight, we used logistic regression and all analysis was done by SPSS 16 software and $P<0.05$ was postulated as the significance level.

\section{Results}

In the case group there were 70 patients; 41 patients were male $(58.6 \%)$ and 29 patients $(41.4 \%)$ were female with mean age of $5.19 \pm 3.24$ years. A total of 140 patients were in the control group after matching for age and gender. Among the 210 participants in this study, $8.6 \%$ were in the case group, $12.9 \%$ were in control group while $6.4 \%$ had LBW (Table 1).

This study revealed that the risk of INS in patients with LBW is two times higher than those with normal birth weight. However, there was no statistically significant difference $\left(\chi^{2}=1.58, P=0.12\right)$ (Table 2$)$.

\section{Discussion}

In this case-control study, evidence for the risk of INS in LBWs was evaluated. Nephrogenesis normally leads to a relatively fixed nephron proportion and if deficits occur during nephrogenesis, nephron development may be interrupted and cause inborn nephron deficit and oligonephronia (3). A variety of factors influence fetal growth categorized as fetal, maternal and placental factors (6).

Birth weight influences a variety of renal parameters particularly proteinuria, renal function and kidney diseases (4). The definition of LBW is variable, however, most authors use the 10th percentile or below. In our study we defined birth weight below $2500 \mathrm{~g}$ for evaluation $(1,6)$. While sub-optimal birth weight may have disadvantages

Table 1. Percent of low birth weight in case and control groups

\begin{tabular}{|c|c|c|c|c|}
\hline & & \multicolumn{2}{|c|}{ Low birth weight } & \multirow{3}{*}{ Total } \\
\hline & & Yes & No & \\
\hline & & No. (\%) & No. (\%) & \\
\hline \multirow{3}{*}{$\begin{array}{l}\text { Syndrome } \\
\text { Nephrotic }\end{array}$} & Yes (Case group) & 9 (12.9) & $61(87.1)$ & 70 (100) \\
\hline & No (Control group) & $9(6.4)$ & 131 (93.6) & 140 (100) \\
\hline & Total & $18(8.6)$ & $192(91.4)$ & $210(100)$ \\
\hline
\end{tabular}

Table 2. Result of logistic regression for infant syndrome nephrotic by low birth weight

\begin{tabular}{lcccccc}
\hline & \multirow{2}{*}{ B } & \multirow{2}{*}{ SE } & P & OR & \multicolumn{2}{c}{$95 \% \mathrm{Cl}$} \\
\cline { 5 - 7 } & & & & & Lower & Upper \\
\hline LBW (1) & 0.764 & 0.496 & 0.124 & 2.148 & 0.812 & 5.680 \\
Constant & -0.744 & 0.155 & 0.000 & & & \\
\hline
\end{tabular}

Abbreviations: OR, odds ratio; SE, standard error; LBW, low birth weight. 
during perinatal, infancy and even adulthood stages $(4,6)$, LBW is one of the main risk factors for infantile morbidity and mortality. The incidence of LBW is increasing in developed countries and abundant evidence supports the association between low birth weight (LBW) and renal dysfunction in humans (4).

The anatomic measurement of infants, children and adults shows a significant inverse correlation between LBW and the proportion of nephrons $(2,4)$. It is possible that intra-uterine growth retardation (IGR) might cause a low nephron number, which is a predisposing factor for hypertension and progressive renal disease $(1,2)$. Later studies showed that LBW is associated with mild to moderate elevation of blood pressure, a reduced number of compensatory hypertrophied glomeruli and lower GFR and higher urine albumin to creatinine ratio $(2,9)$.

It has been shown that LBW is associated with more frequent relapses of nephrotic syndrome in children with minimal change nephrotic syndrome $(4,9)$. After that, some other studies evaluated the course of kidney disease in patients with LBW. For example, in 1998 Zider et al detected an association between an aggravated course of INS and IUGR. They reported a higher rate of steroid dependency and the use of cytotoxic medication and higher number of relapses in a small gestational age (SGA) group (10). Sheu and Chen showed a higher rate of renal biopsy, higher serum lipids, more steroid dependency and higher number of relapse in SGA (3). In the study by $\mathrm{Na}$ et al, steroid resistance and steroid dependency were seen significantly more often in the SGA group (11). In another study, Plank et al found an evidence for an aggravated course of INS in SGA children (1).

While the prevalence of INS was twice in the case group but there was no statistically significant difference between the two groups. Recently, we studied the outcome of patient with INS in LBW, however we could not find any relationship between LBW and course of INS except for age of presentation (lower age of presentation in the LBW group). Therefore, due to the opposite results, we postulated that genetic and nutritional factors can explain these differences. In our study, the higher but not statistically significant rate of INS among LBWs may reflect the influence of the small sample size in our study.

\section{Conclusion}

Although the result of this study does not show any statistically significant relationship between nephrotic syndrome and LBW but the risk of nephrotic syndrome was twice in the case group. In addition, an aggravated course of INS in LBW children has been reported in other studies. We recommend multicenter studies with a larger sample size for better evaluation.

\section{Limitations of the study}

Probably, a larger sample size in a multi-centric national study, improves the accuracy of this study.

\section{Acknowledgements}

The authors are grateful to all who helped in conducting the present research.

\section{Authors' contribution}

NR; study design, research proposal initiation and approval, and manuscript preparation. AS; study design, Research proposal initiation and approval, and manuscript preparation and review of scientific content. MRT; manuscript preparation and review of scientific content. MSS; Data collection and writing the paper. SH; statistical analysis.

\section{Ethical considerations}

Ethical issues (including plagiarism, misconduct, data fabrication, falsification, double publication or submission, redundancy) have been completely observed by the authors.

\section{Conflicts of interest}

The authors declare no competing interests.

\section{Funding/Support}

This study manuscript extracted from MSc thesis (No. 92251) that was supported financially by the deputy of research of Kermanshah University of Medical Sciences.

\section{References}

1. Plank C, Ostreicher I, Dittrich K, Waldherr R, Voiget $\mathrm{M}$, Amann K, et al. low birth weight, but not postnasal weight gain, aggravates the course of nephrotic syndrome. Pediatric Nephrol. 2007;22:1881-9. doi: 10.1681/ASN.200912124810.1007/s00467-007-0597-9.

2. Vikse B, Irgens L, Leivestad T, Hallan S, Inversen B. Low birth weight increase risk for end stage renal disease. J AM Soc Nephrol. 2008;19:151-7. doi:10.1681/ ASN.2007020252.

3. Sheu J, Chen J. Minimal change nephrotic syndrome in children with intrauterine growth retardation. AM J kidney Dis. 2001;37:909-91. doi: 10.1053/ AJKD.2001.23611.

4. Luychx V, Brenner B. The clinical importance of nephron mass. J AM Soc Nephrol. 2010;10:1-13. doi: 10.1681/ASN.2009121248.

5. Deschenes G, Wittner M, Stefano A, Jounier S, Doucet A, Collecting duct is a site of sodium retention in pan. J AM Soc Nephrol. 2001;12:598-601.

6. Bernabe J, Soriano T, Albaladejo R, Juarranz M, Calle M, Martinez D, et al. Risk factors for low birth weight. Eur J Obstet Gynecol Reprod Biol. 2004;116:3-15. doi: 10.1016/J.EJOGRB.2004.03.007.

7. Teeninga N, Schreuder M, Bokenkamp A, Delemarre $\mathrm{H}$, Wijik J. Influence of low birth weight on minimal change nephrotic syndrome in children, including a met analysis. Nephrol Dial Transplant. 2008;23:1615-20. doi: 


\subsection{3/ndt/gfm829.}

8. Ikezumi Y,Suzuki T, Krasawa T, Yamada T, Hasegawa H, Nishimura $\mathrm{H}$, et al. Low birthweight and premature birth are risk factors for podocytopenia and focal segment glomerulosclerosis. Am J Nephrol. 2013;38:149-157.doi: 10.1159/000353898.

9. Seyedzadeh A, Tohidi MR, Nikibakhsh A, Mahmodzade H, Momtaz H, Dalirani R, et al. Survey of the relationship between body birth weight and outcome of idiopathic nephrotic syndrome. Arak Med Univ J. 2013;16:138-44.
10. Zidar N, Avgustin M, Kenda RB, Ferluga D. Unfavorable course of minimal change nephrotic syndrome in children with intrauterine growth retardation. Kidney Int; 1998; 54: 1320-1323. doi: 10.1046/J.15231755.1998.00121.X.

11. Na YW, Yang HJ, Choi JH, Yoo KH, Hong YS, Lee JW, Kim SK. Effect of intrauterine growth retardation on the progression of nephrotic syndrome. AM J Nephrol 2002;22:463-7. doi: 10.1159/000065275.

Copyright $\odot 2018$ The Author(s); Published by Society of Diabetic Nephropathy Prevention. This is an open-access article distributed under the terms of the Creative Commons Attribution License (http://creativecommons.org/licenses/by/4.0), which permits unrestricted use, distribution, and reproduction in any medium, provided the original work is properly cited. 